\title{
COVID-19 What happens in Mildly Obese Children \\ - HoTeckTuak Immune Response \\ An Engineering perspective
}

\author{
Ho Teck Tuak, PhD. \\ Keywords: COVID-19, Mildly Obese Children, HoTeckTuak Immune Response
}

SARS-CoV-2 is a Virus. It continuously replenishes its lipid layer from the excess fats taken from the host. In Adults, the excess fats are more likely plentiful and the replenishment is usually complete. In children who are mildly obese the amount of excess fats may not be enough. So, the replenishment in the Virus is partially complete. Most of the partially replenished Virus will slowly disintegrate. Those that still survive are likely in a deform stage. As the Virus is not whole it is unlikely able to behave as a complete structure and does not have the resources to complete the journey to reach the lung tissue. So, it falls behind and moves slowly and settled where it is for example, near to the entry site at the neck area or along the blood vessel if it is carried along by the blood flow. As it is not a full Virus its presence is revealed and the immune system likely able to do a partial recognition that there are some foreign objects in those areas and attacks it. The Virus will likely eventually be rendered ineffective by the immune system and with the help from existing treatment for inflammation the patient will most likely recover. For such patients, it is likely the bodily excretions will contain a large amount of the Virus fragments.

I refer or name this the HoTeckTuak Immune Response. It is when a Virus or Bacteria is able to survive in an organism for example, human and is not recognized by the immune system, or evade being noticed by the immune system, and as it replenishes itself or mutate and did not complete the process and becomes deform or partially form and not whole, its presence is revealed to the immune system as a foreign body in the area and is unable to evade being noticed by the immune system, and the immune system attacks it. 